\title{
Correlación entre parámetros químicos, cristalográficos y espectroscópicos en la termometría de grafito aplicada a una aureola de contacto del monzogranito de La Soledad (Andes venezolanos)
}

\author{
Correlation between chemical, crystallographic and \\ spectroscopic parameters in graphite thermometry applied to \\ a contact aureole of La Soledad monzogranite (Venezuelan \\ Andes)
}

\author{
K. Reategui ${ }^{1}$, M. Martínez ${ }^{1,2}$ \\ 1 Centro de Geoquímica, Instituto de Ciencias de la Tierra, Facultad de Ciencias, Universidad Central de Venezuela. Apartado \\ Postal 3895, Caracas 1010 A. Email: katya.reategui@ciens.ucv.ve; ORCID ID: http://orcid.org/0000-0003-3920-0643 \\ 2 Facultad de Ciencias de la Ingeniería, Universidad Estatal Península de Santa Elena. Avda. Principal La Libertad, Ecuador. \\ Email: mmartinez@upse.edu.ec; ORCID ID: http://orcid.org/0000-0003-0985-5673
}

\begin{abstract}
RESUMEN
Muestras de grafito provenientes de una aureola de contacto entre filitas de la Asociación Cerro Azul (Paleozoico) y el Monzogranito de La Soledad en Los Andes venezolanos, fueron estudiadas mediante técnicas químicas (\% Carbono inorgánico y distribución isotópica), cristalográficas (DRX) y espectroscópicas (Raman) con el fin de evaluar los cambios en las características del grafito en las cercanías del contacto, correlacionar los diferentes parámetros entre sí, y estimar la temperatura máxima alcanzada por la roca caja durante la intrusión ígnea. La cristalografía del grafito exhibe cambios notables, y tiene lugar una disminución en el contenido del mineral en las inmediaciones del contacto. El $\delta^{13} \mathrm{C}$ alcanzó valores menos negativos en las cercanías al monzogranito, ocasionado por devolatilización; el grafito presente exactamente en el contacto con el plutón pudo experimentar recristalización posterior al evento térmico, con puntual desplazamiento isotópico hacia valores más negativos. El intervalo de valores de grado de grafitización calculados ( $G D=53-80$ ) corresponde a un mineral bien estructurado y con empaquetamiento ordenado. La temperatura máxima durante el evento intrusivo en el contacto fue calculada a través de los parámetros cristalográficos (DRX) como espectroscópicos (Raman) con gran concordancia en ambas técnicas, registrando $528 \pm 16$ y $526 \pm 20^{\circ} \mathrm{C}$ respectivamente. El nivel metamórfico alcanzado por las rocas metapelíticas en la aureola de contacto comprende la Zona de la Cordierita (cordierita + biotita + muscovita) donde el grafito se halla bien cristalizado y en microtextura hexagonal. Factores como la actividad de fluidos y la recristalización posterior de mineral grafitoso inciden en redistribuciones isotópicas posteriores al evento intrusivo, al igual que en la tasa de cambio de cristalinidad del grafito con la temperatura lo que determina una ausencia clara de correlación entre las variaciones isotópicas de ${ }^{13} \mathrm{C}$ en el grafito y la temperatura.
\end{abstract}

Palabras clave: Grafito; Metamorfismo de Contacto; Venezuela; Metapelitas; DRX; Raman; Isótopos de carbono.

Recibido el 13 de enero de 2017; Aceptado el 9 de agosto de 2017; Publicado online el 7 de noviembre de 2017

Citation / Cómo citar este artículo: Reategui, K. \& Martínez, M. (2017). Correlación entre parámetros químicos, cristalográficos y espectroscópicos en la termometría de grafito aplicada a una aureola de contacto del monzogranito de La Soledad (Andes venezolanos). Estudios Geológicos 73(2): e069. https://doi.org/10.3989/egeol.42748.444.

Copyright: () 2017 CSIC. This is an open-access article distributed under the terms of the Creative Commons Attribution-Non Commercial (by-nc) Spain 3.0 License. 


\begin{abstract}
Graphite samples from a metamorphic contact aureole between phyllites of the Cerro Azul Association (Palaeozoic) and La Soledad Monzogranite, in the Venezuelan Andes, were studied by chemical (\% inorganic carbon and isotopic distribution), crystallographic (DRX) and spectroscopic (Raman) techniques in order to assess changes in the graphite in the vicinity of the contact, the correlation between the different parameters, and the determination of the higher temperature reached by the host rock during igneous intrusion. The $\delta^{13} \mathrm{C}$ reached less negative values near the monzogranite, caused by devolatilization; the graphite present just in contact with the pluton experienced retrograde recrystallization, which causes a shift towards more negative values. The calculated degree of graphitization intervals $(G D=53-80)$ corresponds to a well-structured mineral with ordered packaging. The peak metamorphic temperature at the contact was calculated from crystallographic (XRD) and spectroscopic (Raman) parameters with great agreement in both techniques, registering the $528 \pm 16$ and $526 \pm 20{ }^{\circ} \mathrm{C}$ respectively. The metapelitic rocks reached the Cordierite Zone (cordierite + biotite + muscovite) in the contact aureole where the graphite is well ordered and in hexagonal microtexture. Factors such as fluid activity and the subsequent retrograde recrystallization have an effect on isotopic redistributions after the intrusive event, as well as on the crystallinity change rate with the temperature, avoiding a clear correlation between the isotopic variations of ${ }^{13} \mathrm{C}$ in graphite and the temperature.
\end{abstract}

Keywords: Graphite; contact metamorphism; Venezuela; metapelites; XRD; Raman; Carbon isotopes.

\section{Introducción}

El proceso paulatino de grafitización de los sedimentos carbonosos inicia aproximadamente a $200{ }^{\circ} \mathrm{C}$, tan pronto como los procesos diagenéticos cesan y se han perdido los heteroátomos presentes en la materia orgánica, como nitrógeno, oxígeno e hidrógeno; las reacciones de aromatización y reorganización estructural gobiernan el proceso (Cesare \& Maineri, 1999; Martínez \& Escobar, 2016). En el proceso de grafitización, la reorganización estructural procede tanto en los planos aromáticos hexagonales, por incorporación progresiva de unidades aromáticas $\left(\mathrm{L}_{\mathrm{a}}\right)$ como en los planos perpendiculares a éstos, donde incrementa el apilamiento de las láminas desde $2-3$ hasta 40 y mas $\left(\mathrm{L}_{\mathrm{c}}\right)$, junto a un "ajuste" u ordenamiento de las mismas, a medida que incrementa la temperatura; de esta manera el material grafitoso pasa sucesivamente desde un estado amorfo hasta totalmente ordenado, pasando por estados intermedios de apilamiento turbostrático (Kwiecinska \& Petersen, 2004). La respuesta del material carbonoso al gradiente de temperatura es lo que ha permitido que en los últimos años el proceso metamórfico sea descrito en términos mineralógicos, físicos, químicos e isotópicos (French, 1964; Landis, 1971; Luque et al., 2009; Nakamura \& Akai, 2013). El aspecto físico del proceso de grafitización está caracterizado por el incremento de las dimensiones del plano interno (001), del diámetro $\left(\mathrm{L}_{\mathrm{a}}\right)$ de los anillos, del espesor del empaquetamiento (cantidad de láminas o capas de grafeno) $\left(\mathrm{L}_{\mathrm{c}}\right)$ y por la reducción de la distancia interplanar (d) de los cristales de grafito (Tagiri, 1981). Los valores de estos parámetros definen el grado de cristalinidad del material carbonoso, reflejando básicamente el ordenamiento de los átomos de carbono en la estructura cristalina, lo cual puede ser monitoreado por técnicas cristalográficas, tales como difracción de rayos $\mathrm{X}$, espectroscopía Raman y microscopía electrónica; por otra parte, con estas técnicas se han generado ecuaciones que permiten estimar la máxima temperatura de metamorfismo (Tagiri, 1981; Tagiri \& Oba, 1986; Wopenka \& Pasteris, 1993; Wada et al., 1994).

El proceso de grafitización de la materia orgánica desde el ámbito sedimentario original hasta los dominios metamórficos (grafito singenético) ha sido bien estudiado por numerosos autores, así como la forma en que puede seguirse el progreso de la grafitización a partir de variadas técnicas instrumentales (Tagiri, 1981; Luque et al., 1998, 2009; Wada et al., 2004; Ortega et al., 2010). Sin embargo, se reconoce otra variedad de este mineral, producido a partir de la precipitación de carbono sólido a partir de fluidos ricos en este elemento, tales como $\mathrm{CH}_{4}, \mathrm{CO}_{2} \mathrm{y}$ CO. Los depósitos de grafito epigenético son los más cotizados debido a su mayor pureza; normalmente son hallados en rocas de alto grado metamórfico, tales como granulitas, así como rocas ígneas tanto ácidas como ultrabásicas, e igualmente intrusivas o extrusivas (Luque et al., 1998).

Durante el proceso de grafitización, también están presentes los efectos isotópicos cinéticos, como resultado de las diferencias en las energías de enlace 
entre las especies isotópicas. El enlace ${ }^{12} \mathrm{C}-{ }^{12} \mathrm{C}$ es el primero en ser afectado por el esfuerzo termal (Hoefs \& Frey, 1976). Los gases que emergen y contienen carbono, principalmente $\mathrm{CH}_{4}$, deben ser ricos en el isótopo ligero, derivando en un enriquecimiento del isótopo pesado en el residuo (Morikiyo, 1986; Wada et al., 1994). Después que el grafito de origen singenético se ha formado, la composición isotópica de éste puede variar bajo condiciones de equilibrio a través de reacciones de intercambio con compuestos de carbono (principalmente $\mathrm{CH}_{4}$ y $\mathrm{CO}_{2}$ ) en fase fluida, lo que implica la posible aparición de grafito epigenético (Hoefs \& Frey, 1976; Crespo et al., 2005; Luque et al., 2012).

Diversos estudios isotópicos realizados en zonas asociadas a metamorfismo de contacto y metamorfismo regional en litologías calcáreas han logrado establecer la utilidad del fraccionamiento isotópico de carbono entre grafito y calcita, como geotermómetro (Wada et al., 1994; Morikiyo, 1986; Hoefs, 1987; Satish-Kumar \& Wada, 2000; Baumgartner \& Valley, 2001; Deines, 2004). No obstante, en secuencias silicatadas no se conoce bien el comportamiento isotópico a lo largo de la aureola de contacto y su relación con la temperatura de metamorfismo. Dado que la relación $\mathrm{CH}_{4} / \mathrm{CO}_{2}$ depende de la fugacidad de oxígeno en la fase fluida, se cree que el valor de $\mathrm{fO}_{2}$ debe ser el parámetro más importante que afecta la composición isotópica del grafito, al menos en el formado por precipitación a partir de fluidos. Por lo tanto, el cambio en composición isotópica del grafito completamente ordenado, debería ser relativamente pequeño en rocas no-carbonáticas (Hoefs \& Frey, 1976). En rocas pelíticas precámbricas se ha encontrado que el carbono es isotópicamente pesado en las rocas de alto grado metamórfico, y se ha argumentado que durante el metamorfismo fue liberado metano isotópicamente ligero (Hoefs \& Frey, 1976; Morikiyo, 1986). La existencia de metano en fluidos metamórficos ha sido confirmada por el análisis de inclusiones fluidas en algunos minerales metamórficos (Hollister \& Burruss, 1976). En varios estudios se ha observado un incremento hacia valores más positivos de $\delta^{13} \mathrm{C}$ a medida que aumenta el grado metamórfico en rocas no-carbonáticas, pero éste no es progresivo, ya que parece más bien mantenerse constante entre algunos intervalos (Hoefs \& Frey, 1976; Wada et al., 1994). Estos estudios concluyen que las diferencias en los valores isotópicos dependen de la composición química de los fluidos, originados por las reacciones minerales locales, durante el metamorfismo. Sin embargo, otros investigadores no observaron una correlación entre la composición isotópica y el grado metamórfico (Nabelek et al., 2002).

A diferencia de los parámetros isotópicos, los espectroscópicos (Raman) se presentan más precisos al ser empleados como geotermómetros (Beyssac et al., 2002; Wopenka \& Pasteris, 1993). No obstante, es necesario tomar en cuenta detalles propios de la técnica, como el ángulo del láser, heterogeneidad de las partículas, aplicabilidad a diferentes temperaturas, y la preparación de la muestra para el ensayo (Aoya et al., 2010; Hilchie \& Jamieson, 2014).

El propósito de este trabajo es la correlación entre diferentes parámetros químicos $\left(\%\right.$ de carbono, $\left.\delta^{13} \mathrm{C}\right)$ cristalográficos (DRX) y espectroscópicos (Raman) aplicadas al fenómeno de grafitización del material carbonoso en las proximidades de una aureola de metamorfismo de contacto, con la idea de evaluar su utilidad como geotermómetro en rocas silicatadas. En este trabajo se estudió el contacto metamórfico entre el Monzogranito de La Soledad y las filitas de la Asociación Cerro Azul, en los Andes de Venezuela.

\section{Zona de estudio}

La distribución espacial de las diferentes unidades en el Paleozoico Andino es el resultado de los eventos orogénicos y neo-tectónicos, encontrándose en concomitancia facies sedimentarias, metamórficas y formas tectónicas complejas. La sedimentación paleozoica en Venezuela tiene lugar entre dos períodos de orogénesis, el primero, al final del Pre-Cámbrico ( \pm 600 m.a) y el último al final del Pérmico y comienzos del Triásico $( \pm 250 \mathrm{~m} . \mathrm{a})$. Entre ambos episodios se registra otro período orogénico $( \pm 475 \mathrm{~m} . \mathrm{a})$, en el Flanco Sur Andino (González de Juana et al., 1980). La localidad de estudio, ubicada en el occidente de Venezuela, específicamente en el Estado Barinas, se ubica en una quebrada afluente al río Santo Domingo, localizada entre las poblaciones de La Soledad - San Isidro, a lo largo de la carretera Barinitas Santo Domingo, en donde se observa el contacto 
intrusivo entre la cuarzo monzonita de La Soledad y las filitas de la Asociación Cerro Azul (Fig. 1). La edad de las unidades expuestas en la zona se extiende desde el Precámbrico hasta el Cretáceo (Schubert, 1969; Van der Leij et al., 2016).

En el Flanco Sur de la Sierra de Santo Domingo se encuentra la Asociación Cerro Azul, la cual litológicamente consiste en filitas azul-verdoso, esquistos sericíticos laminados grisáceos grafitosos e intercalaciones de cuarcitas impuras. Algunos cuerpos ígneos afloran en el seno de la asociación, entre los cuales están El Granito Cerro Azul y el granito de la Soledad. Con este último, desarrolla un metamorfismo de contacto de la andalucita, y presenta una alteración a rocas corneanas (Schubert, 1969).

Se ha considerado que la depositación de los sedimentos de la Asociación Cerro Azul, ocurrió en un ambiente cercano a la plataforma por debajo del tren de olas, y sufrieron un metamorfismo regional correspondiente a la facies de los esquistos verdes (González de Juana et al., 1980). No se han encontrado fósiles; sin embargo, ha sido correlacionada con algunas de las formaciones

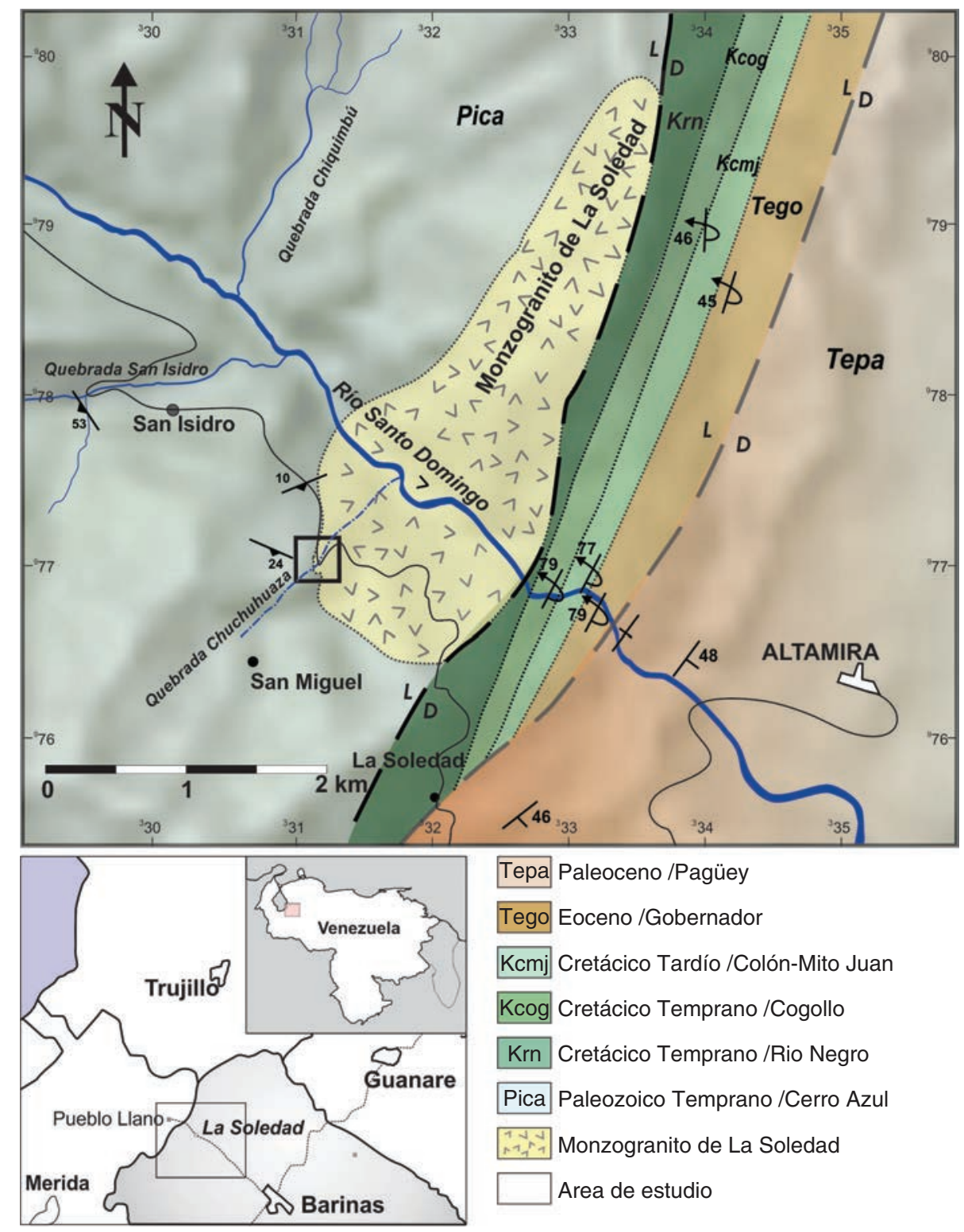

Fig. 1.—Ubicación de la zona de estudio. 
del Paleozoico inferior. El ciclo Paleozoico Inferior se cierra con un período de actividad tectónica, asociado a la parte superior de la orogénesis Caledoniana, marcada por una serie de intrusiones graníticas de edad entre 400-380 m.a. Este período orogénico produjo un largo hiatus durante el Devónico y el Mississipiense (González de Juana et al., 1980).

Algunos estudios ponen en evidencia en la Región Central Andina (Kovisars, 1972; García \& Campos, 1972, entre otros) la presencia de un metamorfismo regional progresivo, asociado a facies de temperatura media a baja presión, correspondiente a los finales del Paleozoico; este episodio afectó, en mayor grado, las zonas este y noreste. Varios eventos graníticos se presentan en la zona Andina, haciéndose progresivamente más jóvenes de sur a norte. En el sureste de los Andes, la actividad granítica se inicia en el Precámbrico Superior-Cámbrico Inferior y los plutones más jóvenes se observan en el Paleozoico Superior, en la porción nor-occidental de la cordillera. Entre estos se encuentra la Cuarzo Monzonita de La Soledad, que intrusiona las filitas de la Asociación Cerro Azul. También, está en contacto de falla con la Formación Río Negro del Cretáceo. Este plutón es de color gris claro, con granos hasta de $5 \mathrm{~mm}$ de diámetro, y con granos porfídicos de feldespato que pueden llegar a medir hasta $2 \mathrm{~cm}$. de longitud. Determinaciones radiométricas de edad por el método potasio-argón, dieron una edad aproximada de 297 m.a. (Schubert, 1969; González de Juana et al., 1980) considera esta edad como indicativa del final del metamorfismo regional de los sedimentos de Cerro Azul, o como edad del levantamiento de esta zona; valores radiométricos de $\mathrm{U} / \mathrm{Pb}$ en el cuerpo granítico indican una edad de cristalización de 475 m.a. (González de Juana et al., 1980). Una datación más reciente $\left({ }^{206} \mathrm{~Pb} /{ }^{238} \mathrm{U}\right.$ en circones) proporciona al monzogranito de La Soledad una edad de 414,5 \pm 3,9 m.a. (Van der Liej et al., 2016).

\section{Muestras y métodos}

\section{Muestreo}

La aureola de contacto se manifiesta a nivel de afloramiento con una extensión de aproximadamente 3 metros. En la zona de muestreo se observa claramente el contacto ígneo-metamórfico (Fig. 3a).
Las muestras fueron tomadas con un espaciamiento promedio de $30 \mathrm{~cm}$, ubicadas paralelas a la aureola de contacto, recolectándose un total de siete muestras. En la zona de contacto, la filita se presenta hornfelizada, con abundantes vetillas de cuarzo de poco espesor ( $1 \mathrm{~mm}-2 \mathrm{~mm})$, orientadas paralelas al contacto pero de forma irregular asemejando fluidos de este mineral, también se observa pirita masiva y en forma de cristales; sin embargo, este mineral no fue observado asociado al grafito, ni a nivel de muestra de mano ni a nivel petrográfico. A los $50 \mathrm{~cm}$. del contacto se observa una veta de cuarzo de 30 a 50 $\mathrm{cm}$. de espesor que se interrumpe en forma de lente; aproximadamente a $80 \mathrm{~cm}$. del contacto empiezan a aparecer porfidoblastos de andalucita que oscilan entre 0,25 y $1,5 \mathrm{~cm}$, sin orientación preferencial, pero dentro de bandas de color gris oscuro (Fig. 2).

La cristalización de andalucita está asociada, generalmente, a temperaturas mayores de $500{ }^{\circ} \mathrm{C}$ y presiones menores a 3 kbar (Turner, 1968; Török, 1992). Debido a que no se observa granate (en ninguna de sus formas), y por la presencia de la textura corneana, propia de presiones menores a $3 \mathrm{kbar}$, la presión asociada a las intrusiones debió estar entre $2-3$ kbar, estimándose que la intrusión se emplazó a bajas profundidades. La conjunción de esta condición de baja presión con temperaturas por encima de los $500^{\circ} \mathrm{C}$ y fluidos con altas concentraciones de Al, Si y O, da origen a la aparición de la andalucita, la cual es característica de zonas de metamorfismo de contacto.

A 1,5 m del contacto se observan bandas de color gris oscuro, de aprox. $2 \mathrm{~cm}$. de espesor, con cristales de andalucita ordenados de aproximadamente $0,3 \mathrm{~cm}$. - 0,4 cm. de longitud; estas bandas se encuentran intercaladas con vetas de cuarzo de $1 \mathrm{~cm}$. de espesor, y zonas de color gris oscuro de grano muy fino. Al sobrepasar esta zona no se observan cristales de andalucita en el afloramiento. En general la filita presenta un color que va de gris claro con brillo mate en las cercanías del contacto, hasta gris oscuro con un brillo sub-metálico, indicando una concentración notable de material grafitoso. La presencia de vetas de cuarzo evidencia actividad de fluidos hidrotermales; por otra parte, la zona de mayor concentración de estas vetas concuerda con la aparición de los cristales de andalucita, lo que permite proponer condiciones físico-químicas diferentes en esta zona. 


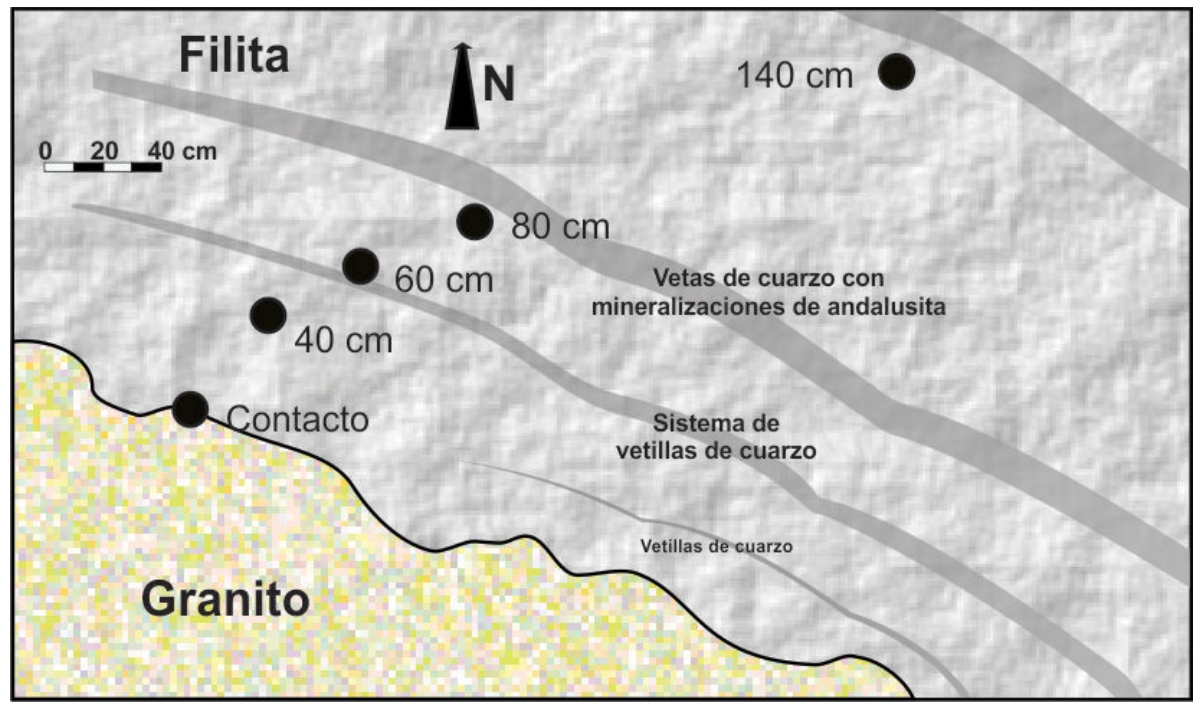

Fig. 2.-Esquema de disposición del contacto granito-filita, las vetas de cuarzo y andalucita, y los puntos de muestreo más cercanos al contacto.

(a)

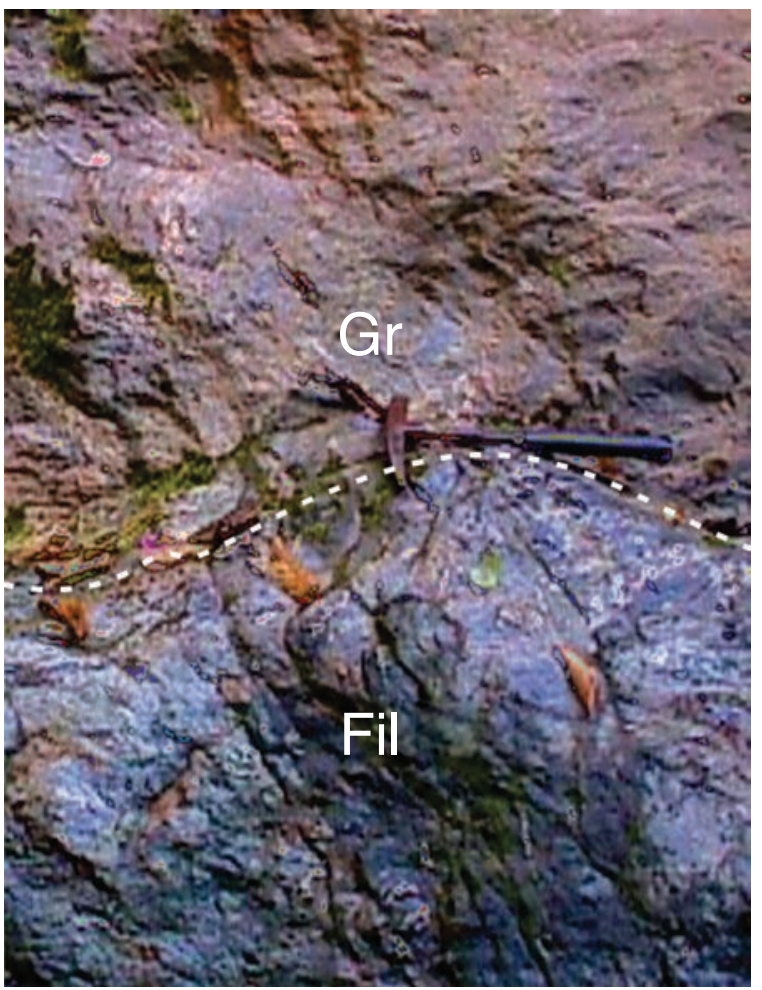

(b)

(c)

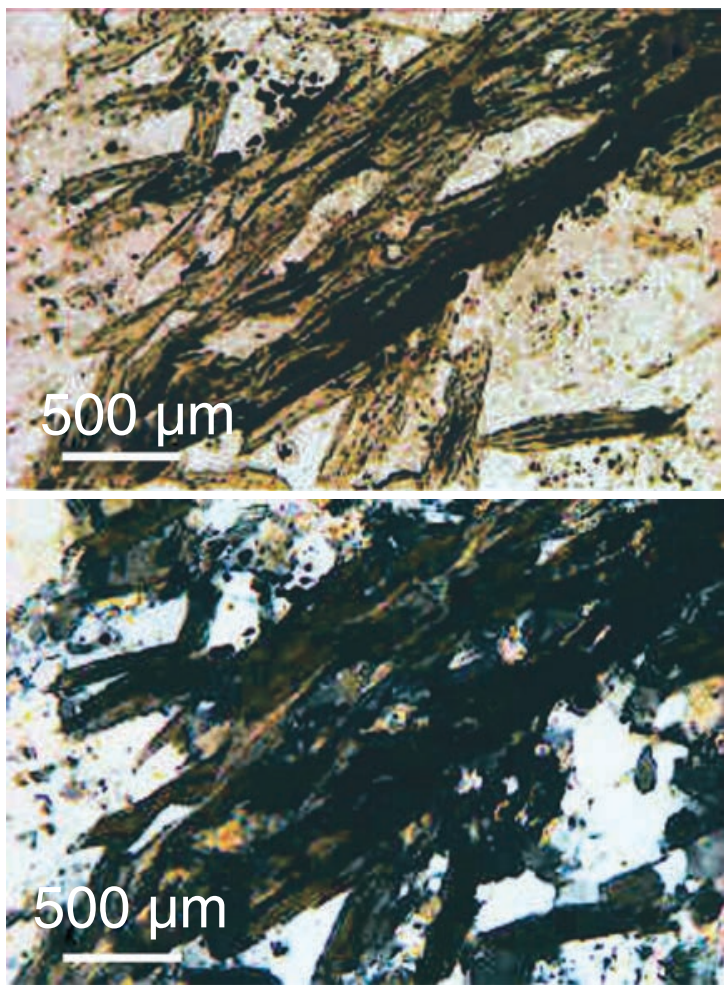

Fig. 3.-(a) Contacto en el campo entre el monzogranito (Gr) y las filitas (Fi) de la Fm. Cerro Azul. (b) Micrografía de muestra de filita a $0,6 \mathrm{~m}$ del contacto. c. Muestra anterior, con luz polarizada. 


\section{Petrografía}

En las muestras más próximas al contacto se observan granos recristalizados de cuarzo de 0,1-0,2 $\mathrm{mm}$. Entre otros minerales recristalizados presentes en esta zona, se observa clorita y moscovita.

En las 3 primeras secciones, pertenecientes a los 0,6 primeros metros del contacto, se observa el material grafitoso en forma asociada, formando cúmulos (Fig. 3b-c), y recristalizado en las zonas de fractura y dentro de los minerales micáceos, característico del grafito asociado a zonas de altas temperaturas y bajas presiones (Pattison, 2006). Se trata de una textura corneana levemente filítica, ya que se observa una muy leve foliación ocasionada por micas tales como moscovita, clorita y biotita. En la muestra tomada a $0,8 \mathrm{~m}$ del contacto, los minerales se encuentran alineados formando vetas; así, el cuarzo forma finas venas, al igual que ocasionales venas de grafito y micas, con la aparición de ocasionales cristales de andalucita quiastolítica; en los puntos donde aparece la andalucita, el grafito se halla en forma dispersa. En algunas secciones se aprecia crenulación y leves sombras de presión asimétricas en los pórfidos de andalucita semirotados, lo que sugiere deformación sincinemática asociada a la intrusión ígnea.

A los 1,4 m siguientes, se observa nuevamente segregación del grafito en el material micáceo pero éste se dispone en forma aleatoria, lo que evidencia una probable recristalización posterior por actividad hidrotermal local; en muestra de mano se observan porfidoblastos de andalucita. En las dos últimas muestras, ubicadas a 4 y $7 \mathrm{~m}$ del contacto, el material grafitoso se encuentra diseminado en la matriz mineral, siguiendo la foliación; la mica predominante es la moscovita. La paragénesis en la zona de la aureola de contacto viene dada por $(\mathrm{Qz}+$ $\mathrm{Pl}+\mathrm{Ms}+\mathrm{Bt}+\mathrm{And}$ ) propia de la facies de Hornfels Hornbléndicos, que corresponde a un intervalo de temperaturas entre 450 y $600^{\circ} \mathrm{C}$ y una presión no mayor a 2 kbar (Tracy \& Frost, 1991).

En la muestra más alejada del contacto el contenido de material grafitoso es alto y al estar disperso en la matriz no permite la distinción de otros minerales. Ya en la región no afectada por la aureola de contacto (muestra de filita no grafitosa colectada en la Quebrada San Isidro, a $2 \mathrm{~km}$ del contacto), la petrografía de las filitas de Cerro Azul revelan un metamorfismo regional que alcanzó condiciones de $\mathrm{T}$ y $\mathrm{P}$ propias de la facies de esquistos verdes, basado en la paragénesis $(\mathrm{Bt}+\mathrm{Ms}+\mathrm{Cl}+\mathrm{Qz})$.

\section{Procedimiento analítico}

Las muestras fueron pulverizadas en forma manual, utilizando mortero de ágata, a fin de minimizar posibles alteraciones térmicas en el grafito; posteriormente fueron tamizadas y cuarteadas. El porcentaje de carbono total e inorgánico se realizó empleando un Analizador columbimétrico de Carbono, UIC Coulometrics. El carbono total es determinado por un sistema de combustión acoplado a celdas electroquímicas. La cantidad de carbono inorgánico se determina mediante la descomposición de los carbonatos en $\mathrm{CO}_{2}$ por la acción del ácido perclórico $1 \mathrm{M}$. La cantidad de carbono grafítico (carbono orgánico), es calculado por la diferencia entre el valor de $\mathrm{C}_{\text {total }} \mathrm{y}$ $\mathrm{C}_{\text {inorgánico. }}$ El error asociado a la determinación del \%C se evaluó mediante la desviación estándar calculada midiendo 5 veces este parámetro en una misma muestra. La veracidad de los datos fue ensayada con patrones internacionales de carbón y carbonato de calcio.

El proceso de enriquecimiento en grafito consistió en una combinación de métodos físicos y químicos, que probó ser más eficiente que los métodos físicos o químicos por separado. La fracción grafitosa se concentró mediante flotación, sometiendo $50 \mathrm{~g}$ de la muestra a ultrasonido, en una mezcla ciclohexano - agua en proporción 1:3 por 20 min., posteriormente se realizó el proceso de extracción en un embudo de separación. En estas condiciones, el grafito se ubica preferencialmente en la interfase agua - ciclohexano, separándose así de la matriz mineral. El extracto se trató con HF (conc.) en una proporción de $10 \mathrm{~mL} / \mathrm{g}$ de muestra por 14 horas; posteriormente el grafito resultante se lavó con agua, se extrajo con un poco de ciclohexano y se secó. Este procedimiento es en esencia, una modificación de los métodos de Castro \& Canto (1992) y Bonijoly et al. (1982).

\section{Análisis por DRX}

Las fracciones enriquecidas en grafito, de cada muestra, se sometieron al análisis de difracción de rayos - X empleando un equipo Siemens D8 
Advanced, utilizando como fuente la $\lambda$ de la señal $\mathrm{K} \alpha$ del $\mathrm{Cu}$. Los espectros fueron recolectados en dos partes: un espectro entre $24^{\circ}-28^{\circ}$, para evaluar los parámetros obtenidos del pico d (002), y un espectro entre $2^{\circ}-60^{\circ}$ para evaluar posibles minerales aún presentes en el extracto grafitoso, así como otras líneas de grafito. En cada ampliación de la señal del grafito, fue medida la longitud de onda en Ångströms al centro del pico, y el ancho de la señal a la semialtura, en gradianes. Los errores asociados a este análisis se determinaron mediante el cálculo de propagación de errores, en cada una de las fórmulas aplicadas (ecuaciones 1, 2 y 3 ) (Wada et al., 1994; Cuesta et al., 1998):

$$
\begin{aligned}
& L c=(K \lambda) /\left(\beta_{(002)} \times \cos \Theta\right) \\
& G D=\left[\left(d_{(002)}-3,7\right) /\left(\log L c_{(002)} / 1000\right)\right] \times 100
\end{aligned}
$$

$$
\begin{gathered}
T\left({ }^{\circ} C\right)=3,2 \times G D+280 \\
\text { Lc }=\text { Espesor del cristal (angstroms) } \\
\mathrm{K}=\text { Constante } \text {, valor utilizado 0,9 (Griffin, } 1967) \\
\lambda=\text { Longitud de onda (angstroms) } \\
\beta_{(002)}=\text { Ancho en gradianes a la mitad de la altura } \\
\quad \text { del pico } \\
\mathrm{GD}=\text { Grado de grafitización } \\
\mathrm{d}_{(002)}=\text { Espaciamiento interplanar }
\end{gathered}
$$

\section{Análisis por Espectroscopía Raman}

Para este análisis se empleó un espectrógrafo Dilor XY, con doble monocromador y un detector SPECTRUM1 CCD, con enfriamiento de nitrógeno; se emplea un microscopio OLYMPUS, el láser empleado es de fuente de Argón iónico (514,5 $\mathrm{nm})$. El tamaño del confocal permite estudiar un área de $1-3 \mu \mathrm{m}$ con un objetivo de $50 \mathrm{X}$, empleando un filtro de intensidad 1 para un poder final del láser menor a $5 \mathrm{~mW}$. La muestra fue colocada sobre un porta muestras y compactada presionándola con otra lámina de vidrio, para obtener un mayor número de cristales perpendiculares al haz. Se estudiaron tres puntos en cada muestra, en la región de $1200-1800 \mathrm{~cm}^{-1}$, para cada espectro se tomaron 3 acumulaciones de $300 \mathrm{~s}$ cada una. El área de los picos y los diferentes parámetros tales como, amplitud e intensidad, fueron calculados empleando el programa Origin 5.0. Los errores asociados a esta determinación fueron evaluados calculando la desviación estándar de cada muestra, para ello en cada muestra se midieron tres puntos, promediando los valores obtenidos. De esta manera se evalúan también las heterogeneidades de la muestra con respecto a la cristalinidad del material grafitoso. La temperatura máxima de metamorfismo se evaluó con las ecuaciones 4 y 5 , propuestas por Beyssac et al. (2002).

$$
R 2=D 1 /(G+D 1+D 2)
$$

$T\left({ }^{\circ} \mathrm{C}\right)=-445 \times R 2+641$

\section{$\mathrm{R} 2=$ Relacion de areas entremodos vibracionales}

$\mathrm{G}=$ Mododevibracion a $1580 \mathrm{~cm}^{-1}$

$\mathrm{D} 1=$ Modo vibracional $1350 \mathrm{~cm}^{-1}$

$\mathrm{D} 2=$ Modo vibracional a $1620 \mathrm{~cm}^{-1}$

\section{Análisis isotópicos}

La determinación de la distribución isotópica de carbono-13 se efectuó mediante espectrometría de masas de relación isotópica en flujo continuo ("CF-IRMS" por sus siglas en inglés). Cada muestra fue llevada a combustión total en un analizador elemental marca Carlo Erba modelo 1108 y el $\mathrm{CO}_{2}$ producido se transfirió en flujo continuo a un espectrómetro de masas de relaciones isotópicas marca Micromass modelo Isochrom. Se utilizó aproximadamente 0,1 miligramo de muestra en cada combustión. Como estándares se emplearon NBS-22 (aceite) y sacarosa IAEA-CH6.

\section{Resultados}

\section{Análisis de \% C y $\delta^{13} \mathrm{C}$}

Los valores de contenido de carbono total obtenidos en las muestras analizadas corresponden exclusivamente a grafito, debido a la ausencia de carbonatos en las muestras (Tabla 1). Estos valores oscilan entre 0,4 y $2,3 \%$ aproximadamente. 
La distribución de grafito en función de la distancia al contacto muestra una disminución gradual en las cercanías del mismo (primeros cuatro metros), lo que permite establecer el valor original de carbono en la roca alrededor del 2\% (Fig. 4).

Los valores isotópicos de carbono-13 oscilan alrededor de $-28 \%$. La figura 5 exhibe la distribución de $\delta^{13} \mathrm{C}$ en función de la distancia al contacto; con el incremento de la cercanía al plutón granítico, el grafito se vuelve isotópicamente más pesado.

El grafito presente exactamente en el contacto con el plutón presenta una distribución isotópica levemente más liviana que la exhibida por la tendencia general (Fig. 5). En general esta muestra presenta anomalías no sólo en $\delta^{13} \mathrm{C}$ sino también en el contenido de carbono (Fig. 4) y en los parámetros cristalinos y espectroscópicos a determinar posteriormente.

La correlación entre $\delta^{13} \mathrm{C}$ y el contenido de carbono en la roca $(\% \mathrm{C})$ es presentada en la figura 6. Aunque la correlación no es lineal, se aprecia con

Tabla 1.-Porcentaje de carbono total y composición isotópica de las muestras de la Asociación Cerro Azul

\begin{tabular}{|c|c|c|}
\hline Distancia (M) & $\begin{array}{c}\text { CARBono } \\
(+/-0,10 \%)\end{array}$ & $\begin{array}{l}\Delta{ }^{13} \mathrm{C}_{\mathrm{VPDB}} \\
(+/-0,2 \%)\end{array}$ \\
\hline 0,0 & 0,56 & $-27,5$ \\
\hline 0,40 & 0,39 & $-26,3$ \\
\hline 0,60 & 0,52 & $-27,1$ \\
\hline 0,80 & 1,00 & $-27,2$ \\
\hline 1,40 & 0,66 & $-27,5$ \\
\hline 4,00 & 2,26 & $-27,7$ \\
\hline 7,00 & 1,83 & $-28,3$ \\
\hline
\end{tabular}

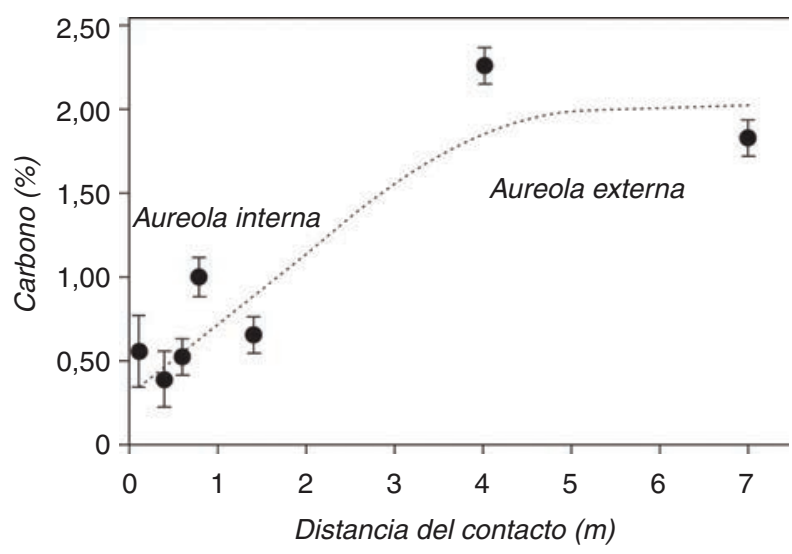

Fig. 4.-Distribución del \%C en la aureola de contacto La Soledad - Cerro Azul. claridad un incremento en la relación isotópica ${ }^{13} \mathrm{C} /{ }^{12} \mathrm{C}$ con la disminución del contenido de carbono, lo que permite proponer que la fracción liberada por devolatilización (esencialmente $\mathrm{CH}_{4}$ por reacción de grafito con el vapor de agua, Pattison, 2006) está enriquecida en ${ }^{12} \mathrm{C}$; sin embargo, podría estarse formando también $\mathrm{CO}_{2}$ originado por la reacción entre el grafito y otras fases minerales (Hoefs \& Frey, 1976; Morikiyo, 1986).

\section{Análisis por DRX}

En la Tabla 2 se muestran los parámetros obtenidos $\mathrm{y}$ calculados del análisis de difracción de rayos $\mathrm{X}$. Los espectros tomados entre $24-28^{\circ}$ son muy similares para todas las muestras, reflejando principalmente diferencias en el ancho de la banda (002), lo cual se asocia al espesor del empaquetamiento $\left(\mathrm{L}_{\mathrm{c}}\right)$.

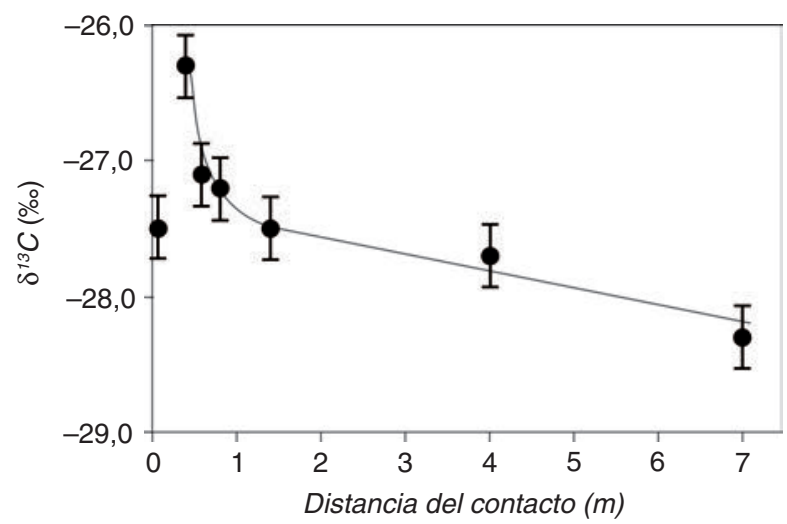

Fig. 5.-Distribución de $\delta^{13} \mathrm{C}$ en la aureola de contacto La Soledad - Cerro Azul.

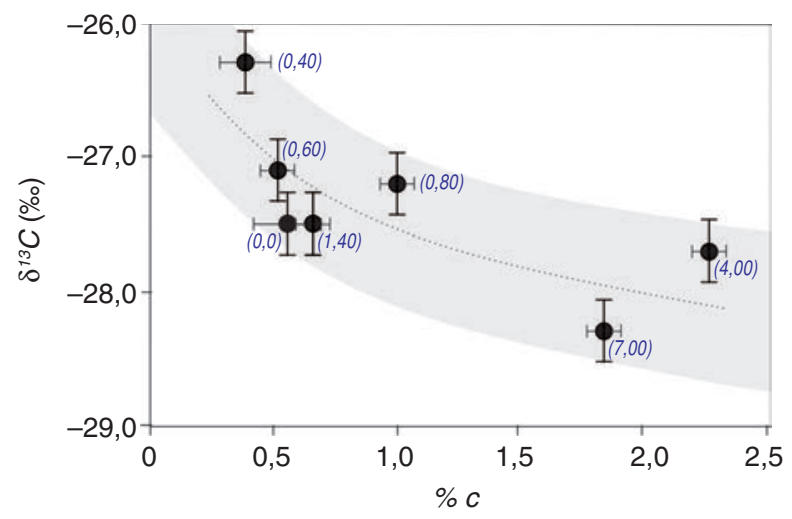

Fig. 6.-Correlación entre el \%C y los valores de $\delta^{13} \mathrm{C}$. La región sombreada en el gráfico ilustra la región de incertidumbre analítica de los parámetros. Los números en paréntesis corresponden a la distancia al contacto, en metros. 
Tabla 2. Parámetros cristalográficos extraídos del análisis por DRX. $\Delta$ : desviación, calculada por propagación de errores. $d=$ Distancia interplanar; $\beta=$ Ancho a la semialtura del pico de DRX; Lc= Espesor del empaquetamiento de láminas de grafito; $\mathrm{GD}=$ Grado de Grafitización; T= Temperatura.

\begin{tabular}{lccccccccc}
\hline distancia $(\boldsymbol{m})$ & $\boldsymbol{d}(\boldsymbol{A})$ & $\boldsymbol{\Delta} \boldsymbol{d}(\boldsymbol{A})$ & $\boldsymbol{\beta}\left({ }^{\circ}\right)$ & $\boldsymbol{L c}(\boldsymbol{A})$ & $\boldsymbol{\Delta} \boldsymbol{L c}(\boldsymbol{A})$ & $\boldsymbol{G D}$ & $\boldsymbol{\Delta} \boldsymbol{G D}$ & $\boldsymbol{T}\left({ }^{\circ} \boldsymbol{C}\right)$ & $\boldsymbol{\Delta} \boldsymbol{T}\left({ }^{\circ} \boldsymbol{C}\right)$ \\
\hline 0,0 & 3,3664 & 0,0006 & 0,36 & 233 & 5 & 53 & 1 & 449 & 10 \\
0,4 & 3,3621 & 0,0004 & 0,26 & 376 & 7 & 80 & 1 & 535 & 10 \\
0,6 & 3,3650 & 0,0005 & 0,26 & 365 & 8 & 77 & 2 & 525 & 12 \\
0,8 & 3,3642 & 0,0004 & 0,27 & 328 & 6 & 69 & 1 & 502 & 10 \\
1,4 & 3,3628 & 0,0005 & 0,24 & 367 & 8 & 77 & 2 & 528 & 12 \\
4,0 & 3,3631 & 0,0004 & 0,27 & 331 & 6 & 70 & 1 & 505 & 10 \\
7,0 & 3,3658 & 0,0004 & 0,30 & 287 & 5 & 62 & 1 & 477 & 9 \\
\hline
\end{tabular}

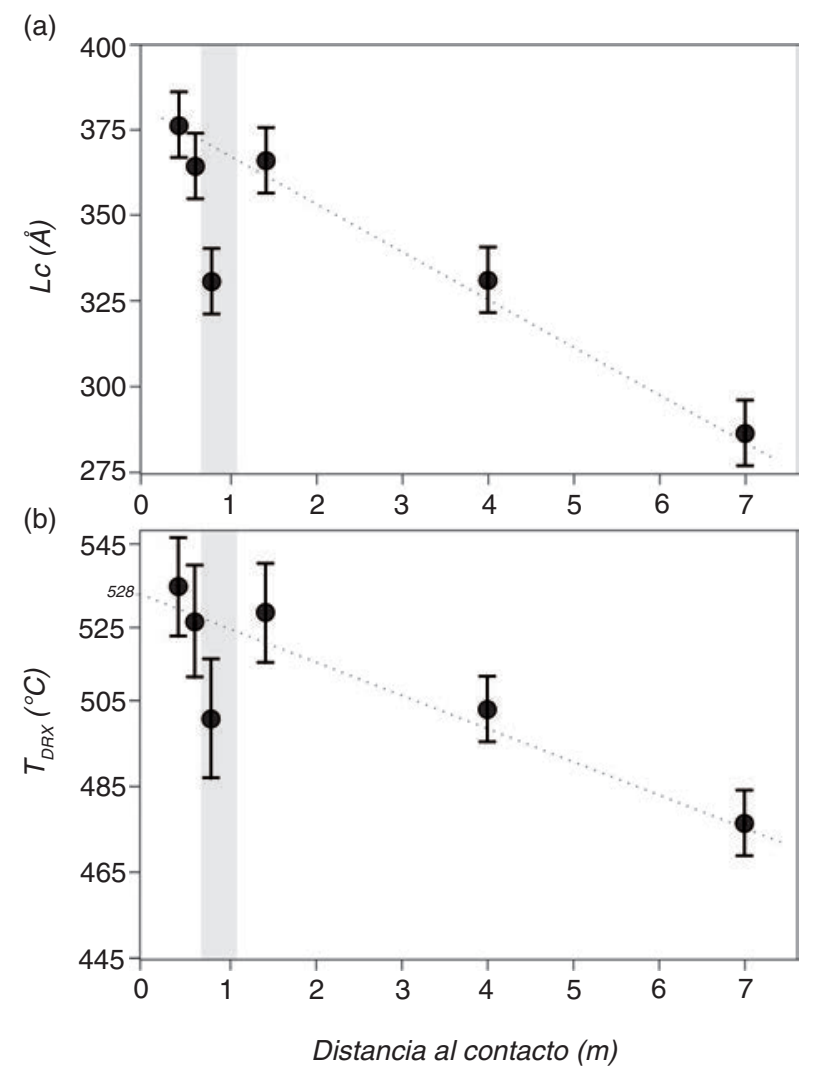

Fig. 7.-(a) Distribución del parámetro cristalino Lc del grafito, calculado a partir de DRX, en función a la distancia del contacto La Soledad - Cerro Azul. (b) Temperaturas calculadas según Wada et al. (1994). La franja gris corresponde a la zona mineralizada con cuarzo y andalucita.

En general, según clasificaciones anteriores (French, 1964; Landis, 1971; Tagiri, 1981), los valores de $\mathrm{d}(002)$ corresponden a un grafito bien ordenado y varían muy poco a lo largo de la aureola. Por el contrario, el parámetro $L_{c}$ sí presenta mayor variación entre las muestras de esta zona, incrementándose a medida que la distancia al contacto disminuye (Fig. 7), siendo en promedio mayores a $300 \AA$.
Asímismo, los valores del grado de grafitización (GD) se asocian a un avanzado proceso de reordenamiento del grafito, proporcionando un valor promedio de 70 (53-80).

La muestra más cercana al contacto no fue considerada en la evaluación de los parámetros cristalográficos, a causa del reducido contenido en carbono y la recristalización retrógrada, lo que generó errores importantes durante el aislamiento del material grafitoso y posterior análisis por DRX.

A partir de la ecuación propuesta por Wada et al. (1994) (ecuación 3), para litologías pelíticas asociadas a terrenos de metamorfismo regional, fue calculada la temperatura máxima alcanzada para cada muestra; los valores obtenidos y las variaciones entre muestras a lo largo de la aureola se presentan en la figura $7 \mathrm{~b}$. La temperatura máxima calculada a través de esta ecuación (muestra a 40 $\mathrm{cm}$ del contacto) es de $535 \pm 10{ }^{\circ} \mathrm{C}$; en contraste, la temperatura más baja se alcanza para la muestra más alejada del contacto, $477^{\circ} \mathrm{C}$. La temperatura calculada por extrapolación de la tendencia de valores hasta el contacto arroja un valor de $528 \pm$ $16^{\circ} \mathrm{C}\left(\mathrm{r}^{2}=0,7265\right)$.

\section{Análisis por Espectroscopía Raman}

En la Tabla 3 se muestran los parámetros obtenidos del análisis de espectroscopía Raman. Los valores de R2, la forma de los espectros y la frecuencia de aparición de los picos característicos coinciden con lo establecido por diferentes autores (Wopenka \& Pasteris, 1993; Beyssac et al., 2002; Aoya et al., 2010) como asociados a materiales grafitosos altamente ordenados, de alto grado metamórfico.

En general, la forma de los espectros se mantiene muy similar para todas las muestras de esta zona. 
Tabla 3. Valores promedios de los parámetros calculados por Raman. $\Delta$ : desviación, calculada por propagación de errores.

\begin{tabular}{lcccccc}
\hline Distancia $(\boldsymbol{m})$ & ID1/IG & $\boldsymbol{\Delta}$ (ID1/IG) & $\boldsymbol{R 2}$ & $\boldsymbol{\Delta} \boldsymbol{R 2}$ & $\boldsymbol{T}\left({ }^{\circ} \mathbf{C}\right)$ & $\boldsymbol{\Delta} \boldsymbol{T}\left({ }^{\circ} \mathbf{C}\right)$ \\
\hline 0,0 & 0,137 & 0,033 & 0,223 & 0,030 & 542 & 13 \\
0,4 & 0,175 & 0,051 & 0,280 & 0,030 & 516 & 13 \\
0,6 & 0,180 & 0,043 & 0,272 & 0,036 & 520 & 16 \\
0,8 & 0,160 & 0,042 & 0,252 & 0,044 & 529 & 20 \\
1,4 & 0,153 & 0,021 & 0,256 & 0,018 & 527 & 8 \\
4,0 & 0,163 & 0,077 & 0,264 & 0,081 & 524 & 36 \\
7,0 & 0,194 & 0,021 & 0,294 & 0,010 & 510 & 5 \\
\hline
\end{tabular}
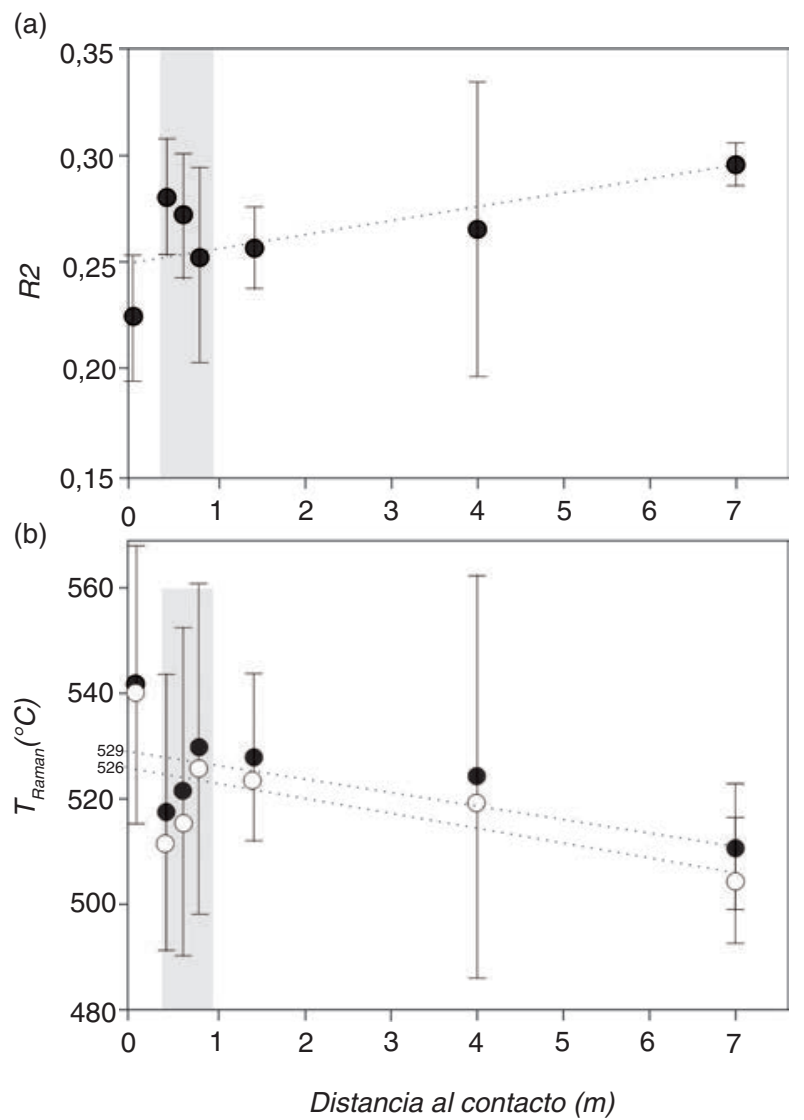

Fig. 8.-(a) Distribución del parámetro R2 calculado por Raman en función a la distancia del contacto La Soledad - Cerro Azul. (b) En círculos negros, temperaturas calculadas según Beyssac et al. (2002); en círculos blancos, según Aoya et al. (2010). La franja gris corresponde a la zona mineralizada con cuarzo y andalucita.

En la figura 8a se muestra la distribución de la relación de áreas R2, a medida que aumenta la distancia al contacto; los valores de R2 disminuyen en las cercanías del contacto, lo que está relacionado con una proporción de orden/desorden mayor, es decir, las muestras en las cercanías del contacto se encuentran más ordenadas.
El error presente en la determinación de los parámetros en cada muestra está asociado a las heterogeneidades del material grafitoso presente en la misma, o a la orientación de los granos de grafito. Para evaluar en mejor grado este parámetro se debe medir por lo menos 30 puntos en una misma muestra (Aoya et al., 2010). Por otra parte, el empleo de los concentrados de grafito en el análisis por Raman pudo incidir negativamente en la expresión del error.

Las temperaturas para cada muestra se determinaron empleando la ecuación originada por Beyssac et al. (2002) utilizando el valor de R2 (Fig. 8b). La temperatura calculada con esta ecuación en la muestra ubicada en el contacto fue de $542^{\circ} \mathrm{C} \pm 15^{\circ} \mathrm{C}$ siendo la temperatura de la muestra más alejada del contacto es de $510^{\circ} \mathrm{C}$. La extrapolación de la línea de tendencia (Fig. 7b) arroja un valor de $529 \pm 20^{\circ} \mathrm{C}$. En las gráficas obtenidas por los estudios de Raman se observa, al igual que en los estudios de DRX, zonas de alta dispersión en los parámetros de R2 y temperatura; coincidiendo, asímismo, con las zonas en donde están presentes los cristales de andalucita y una alta concentración de vetas de cuarzo.

Como se trata de metamorfismo de contacto, fue ensayada la ecuación 6, propuesta por Aoya et al. (2010), quienes observaron una diferencia reproducible entre muestras provenientes de metamorfismo regional y metamorfismo de contacto.

$$
T\left({ }^{\circ} C\right)=221,0(R 2)^{2}-637,1(R 2)+672,3
$$

Haciendo uso de esta ecuación, la temperatura máxima alcanzada es de $541^{\circ} \mathrm{C}$, mientras que la muestra más alejada arroja un valor de $504^{\circ} \mathrm{C}$. La línea de tendencia para los datos de temperatura calculados con la ecuación de Aoya et al. (2010) indica que para el 


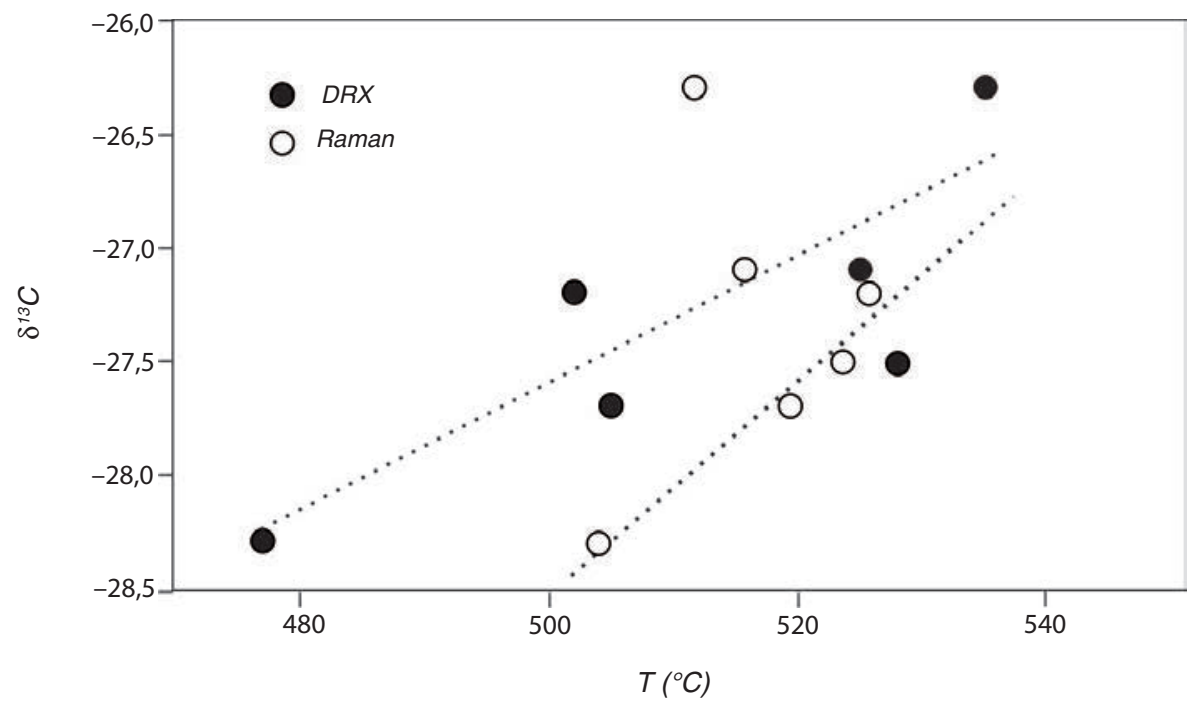

Fig. 9.-Correlación de las temperaturas calculadas por DRX (círculos negros) y Raman (círculos blancos) con los datos isotópicos. Por razones de claridad, se han omitido las barras de error.

contacto, la temperatura máxima es de $526 \pm 20^{\circ} \mathrm{C}$. Las diferencias entre los resultados de ambas ecuaciones son poco significativas, tomando en cuenta que son mucho menores que el error asociado a los valores calculados.

En la figura 9 se presenta la relación entre los gradientes de temperaturas metamórficas, determinadas por técnicas de DRX y Raman para esta zona, y los valores de $\delta^{13} \mathrm{C}$. No se observa una clara correlación entre la temperatura determinada por DRX y Raman con los valores isotópicos; la pendiente de las líneas de regresión son positivas lo que indica que tiene lugar un aumento en los valores de $\delta^{13} \mathrm{C}$ al aumentar la temperatura metamórfica. Esto corrobora lo expuesto anteriormente y concuerda con estudios previos (Hoefs \& Frey, 1976; Morikiyo, 1986). Sin embargo, la ausencia de una linealidad clara permite proponer que la variación isotópica experimentada por el grafito no está solo relacionada con la temperatura, sino a la actividad de fluidos ígneos, la presencia de ciertas fases minerales y posibles recristalizaciones post-metamórficas.

Un indicio de la presencia de tales fenómenos deriva del análisis estadístico de la correlación entre las temperaturas máximas calculadas por ambas técnicas: el Coeficiente de correlación de Pearson calculado para las muestras arroja un valor bajo y negativo $(-0,46)$; sin embargo, al retirar del conjunto de datos la muestra anómala del contacto, donde está evidenciado claramente tanto por isótopos como por Raman que fue afectada por actividad de fluidos, el mismo coeficiente alcanza un valor de $+0,91$.

\section{Discusión}

\section{Distribución isotópica}

La distribución isotópica de carbono-13 en el grafito estudiado corresponde a material carbonoso de origen biogénico (Hoefs \& Frey, 1976). El incremento en este parámetro en las cercanías del contacto es ocasionado por el intercambio isotópico entre el grafito y las fases fluidas, concomitante con la devolatilización que tiene lugar en las condiciones imperantes durante la intrusión (Luque et al., 2012).

La muestra isotópicamente más liviana que rompe la tendencia anteriormente descrita y ubicada exactamente en el contacto también muestra valores muy bajos en el contenido de carbono (Fig. 4), así como en los parámetros cristalinos y espectroscópicos (Figs. 3, 6 y Tabla 2); esta diferencia sugiere la existencia de sobrecrecimiento y/o recristalización retrógrada del material grafitoso (Satish-Kumar \& Wada, 2000).

Por otra parte, la relación hallada en la figura 6 , que revela el incremento en la relación isotópica ${ }^{13} \mathrm{C} /{ }^{12} \mathrm{C}$ con la disminución del contenido de carbono, es cónsona con la liberación de volátiles isotópicamente livianos (esencialmente $\mathrm{CH}_{4}$ por reacción de grafito con el vapor de agua del cuerpo granítico, Pattison, 2006). No se descarta también la posible formación de $\mathrm{CO}_{2}$ originado por la reacción entre el grafito y otras fases minerales (Hoefs \& Frey, 1976; Morikiyo, 1986). 


\section{Cristalinidad del grafito}

La información derivada de las determinaciones por DRX permite proponer que el material carbonoso original presente en la roca antes de la intrusión, era un grafito ya bien ordenado; sin embargo, el proceso termal propició un mayor ordenamiento en las capas de grafeno, aumentando el espesor de empaquetamiento $\left(\mathrm{L}_{\mathrm{c}}\right)$, y las dimensiones del cristal de grafito en el plano basal $\left(\mathrm{L}_{\mathrm{a}}\right)$.

Aun cuando las ecuaciones de Wada et al. (1994) han sido empleadas generalmente en casos donde tiene lugar grafitización progresiva derivada de metamorfismo regional o de contacto, y no fueron pensadas para grafito precipitado a partir de fluidos, en el presente estudio se ha documentado su aplicación exitosa en una aureola de contacto parcialmente afectada por actividad hidrotermal. Este resultado permite proponer que la movilización de grafito a causa de los fluidos hidrotermales tuvo sólo una extensión limitada.

\section{Temperatura en el contacto}

La disminución de temperaturas a través de la aureola estudiada no es gradual, fenómeno que también se observa en la distribución de los parámetros cristalográficos. La zona de mayor dispersión en la gráfica presentada en la figura 8 y señalada en una franja gris alrededor de un metro del contacto, coincide con la aparición de mineralizaciones de andalucita y vetillas de cuarzo. Este hecho permite proponer cierto grado de actividad hidrotermal que afectó localmente la distribución de calor y por tanto, la grafitización del material carbonoso; explicaría igualmente la recristalización o sobrecrecimiento retrógrado en el grafito ubicado exactamente en el contacto ígneo. Este fenómeno no obstante, no es reflejado en los valores isotópicos (Fig. 5).

La figura 10 permite establecer de forma clara que las muestras de roca en la zona del contacto ígneo estudiado se hallan en la llamada Zona de la

(a)

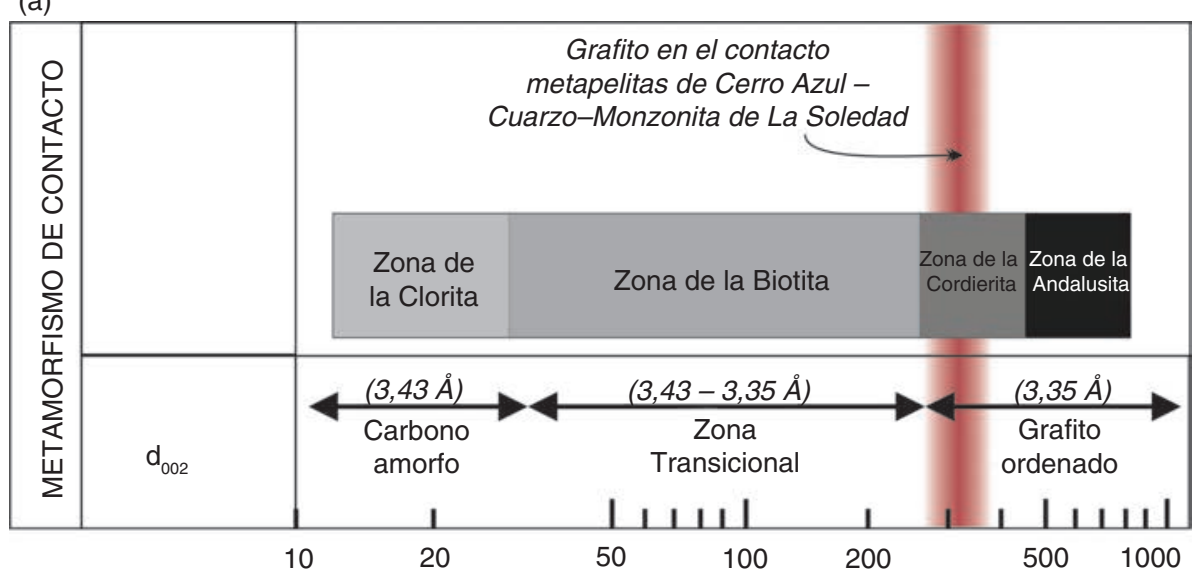

(b) $\quad L_{c}(\AA)$

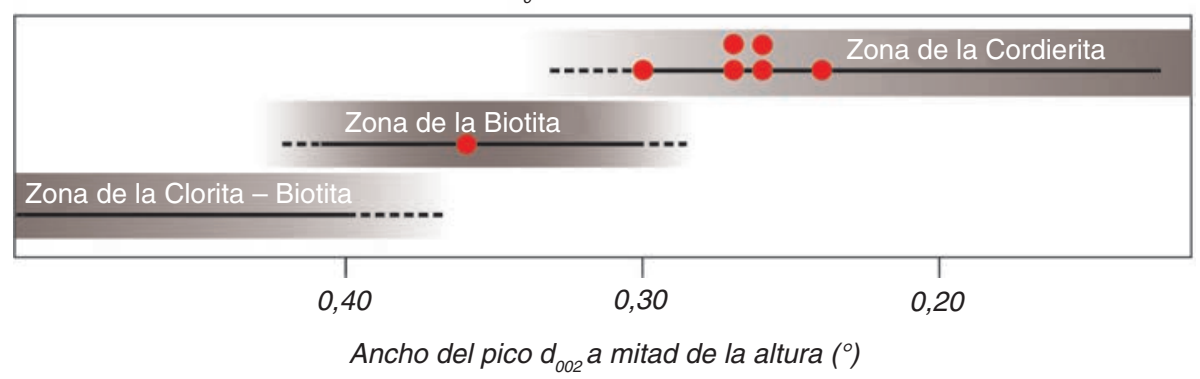

Fig. 10.-(a) Correlación entre el parámetro cristalino Lc y las zonas mineralógicas en metamorfismo de contacto. Para el contacto estudiado, las rocas metapelíticas de la Asociación Cerro Azul alcanzaron la zona de la cordierita (modificado de Nakamura \& Akai 2013).(b) Ubicación de las muestras de la aureola estudiada en las zonas metamórficas acorde con el ancho de la señal $d_{002}$ del grafito (Nakamura 1995). 


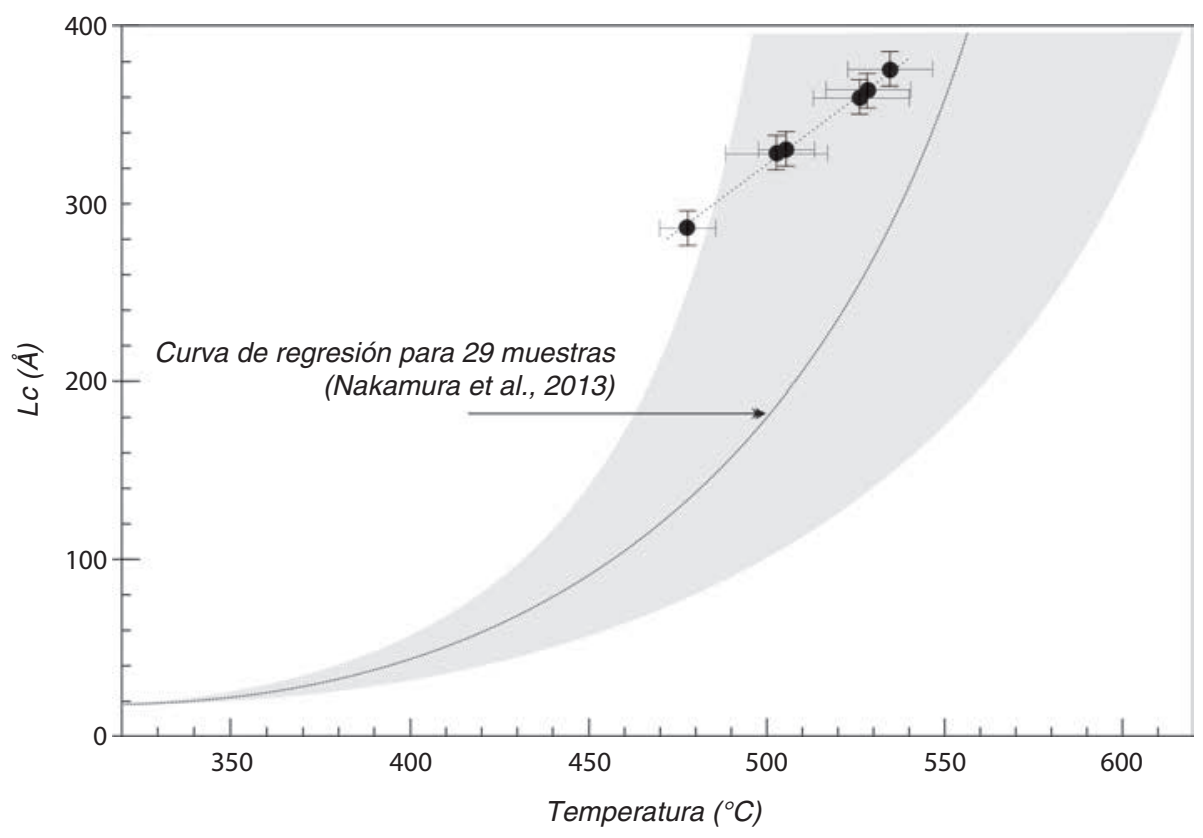

Fig. 11.- Relación entre microtextura y tamaño del cristal (Lc) con la temperatura calculada según Aoya et al. (2010) para la aureola de contacto del Monzogranito de La Soledad (círculos negros). Se incluye con fines comparativos, la tendencia en 29 muestras de la aureola de contacto de Gyoja-yama (zona sombreada, Nakamura \& Akai, 2013).

Cordierita (temperaturas superiores a $500^{\circ} \mathrm{C}$ y presión menor o igual a $3 \mathrm{kbar})$.

\section{Correlación entre temperatura y distancia del contacto}

La tendencia o correlación entre la temperatura calculada por DRX y el $\mathrm{L}_{\mathrm{c}}$ es representada en el diagrama propuesto por Nakamura \& Akai (2013) (Fig. 11), elaborada a partir de los valores obtenidos para la aureola de contacto de la Granodiorita Gyoja-yama, de edad Cretácica, al noroeste de Kyoto en Japón. La aureola de contacto estudiada corresponde a la transición desde la parte final de la zona de la Biotita (ensamblaje de biotita + muscovita + plagioclasa + feldespato-K + cuarzo) a la zona de la cordierita, gobernada por el ensamblaje mineralógico cordierita + biotita + muscovita $(+$ plagioclasa + feldespato potásico + cuarzo). La aureola del Monzogranito de La Soledad sigue una ruta de enfriamiento diferente a la mostrada por la aureola de la Granodiorita de Gyoja-yama; la pendiente de variación $\left(\delta \mathrm{L}_{\mathrm{c}} / \delta \mathrm{T}\right)$ calculada para La Soledad es de $1,667 \AA /{ }^{\circ} \mathrm{C}$; mucho menor que la derivada de la curva de tendencia calculada a partir de la gráfica de Nakamura \& Akai (2013), 5,21 $\AA /{ }^{\circ} \mathrm{C}$. Este resultado permite proponer diferencias notables en las condiciones fisicoquímicas asociadas al enfriamiento de ambos plutones. La menor tasa de cambio en la cristalinidad del grafito con la temperatura no depende de manera significativa de la velocidad de enfriamiento (Nakamura, 1995); de modo que otros factores, como la presencia de fluidos hidrotermales, pudieron afectar el proceso de grafitización.

\section{Conclusiones}

1. La concentración de grafito en las metapelitas de la Asociación Cerro Azul en la aureola de contacto con el Monzogranito de La Soledad disminuye en forma paulatina desde aproximadamente $2 \%$ al inicio en la roca a 7 metros del intrusivo, hasta $0,39 \%$ en el contacto.

2. El grafito cercano al contacto se encuentra muy bien estructurado, como queda determinado por los valores de $\mathrm{d}(002)$ (add a parenthesis) $2 \leq$ 3,366 y de $\mathrm{L}_{\mathrm{c}} \geq 300$.

3. Varios factores afectan la variación en grafito y el $\delta{ }^{13} \mathrm{C}$ en la aureola de contacto estudiada, siendo determinantes, además de la temperatura producto de la intrusión, la presencia y composición de los fluidos presentes, las 
posibles reacciones de éstos con las diferentes facies minerales y el sobrecrecimiento por recristalización del grafito en procesos posteriores.

4. Existe una excelente correlación entre la temperatura máxima calculada por DRX $\left(528 \pm 16^{\circ} \mathrm{C}\right)$ y por Espectroscopía Raman $\left(526 \pm 20{ }^{\circ} \mathrm{C}\right)$ para la aureola de contacto. Esta concordancia entre técnicas independientes permite proponer que el crecimiento basal del cristal $\left(\mathrm{L}_{\mathrm{a}}\right)$, determinado por Raman, tuvo lugar en forma concomitante con el incremento en el espesor del cristal de grafito $\left(\mathrm{L}_{\mathrm{c}}\right)$, determinado por DRX.

5. El intrusivo generó un desplazamiento de $\delta^{13} \mathrm{C}$ hacia valores más positivos en las cercanías del contacto, a causa del gradiente de temperatura. La ausencia de linealidad clara o de una correlación definida entre este parámetro y DRX o Raman, no permite emplear los valores de $\delta^{13} \mathrm{C}$ como geotermómetro en este tipo de ambiente geológico. Sin embargo, es de utilidad en la detección de otros fenómenos asociados al contacto, como la acción de fluidos hidrotermales o sobrecrecimiento del grafito post-intrusión.

\section{AGRADECIMIENTOS}

Este trabajo fue financiado por el Fondo Nacional para la Ciencia, Tecnología e Innovación (FONACIT) a través del proyecto S1-98003208. Los comentarios aportados por los revisores, Dres. J. Barrenechea y T. Hoeger ayudaron a mejorar notablemente el manuscrito original.

\section{Referencias}

Aoya, M.; Kouketsu, Y.; Endo, S.; Shimizu, H.; Mizukami, T. \& Nakamura, D. (2010). Extending the applicability of the Raman carbonaceous-material geothermometer using data from contact metamorphic rocks. Journal of Metamorphic Geology, 28: 895-914. https://doi.org/10.1111/j.1525-1314.2010.00896.x

Baumgartner, L. \& Valley, J. (2001). Stable isotope transport and contact metamorphic fluid flow. Reviews in Mineralogy and Geochemistry, 43: 415-467. https:// doi.org/10.2138/gsrmg.43.1.415

Beyssac, O.; Rouzaud, J.; Goffé, B.; Brunet, F. \& Chopin, C. (2002). Raman spectra of carbonaceous material in metasediments: a new geothermometer. Journal of Metamorphic Geology, 20: 859-871. https://doi. org/10.1046/j.1525-1314.2002.00408.x
Bonijoly, M.; Oberlin, M. \& Oberlin, A. (1982). A possible mechanism for natural graphite formation. International Journal of Coal Geology, 1: 283-312. https://doi.org/10.1016/0166-5162(82)90018-0

Castro, M. \& Canto, M. (1992). An ultrasonic method for the separation of carbonaceous material from schist for the determination of graphitization degree by X-ray diffraction. Chemical Geology, 100: 191-199. https://doi.org/10.1016/0009-2541(92)90112-I

Cesare, B. \& Maineri, C. (1999). Fluid-present anatexis of metapelites at El Joyazo (SE Spain): Constraints from Raman spectroscopy of graphite. Contributions to Mineralogy and Petrology, 135: 41-52. https://doi. org/10.1007/s004100050496

Cuesta, A.; Dhamelincourt, P.; Laureyns, J.; Martínez, A. \& Tascón, J. (1998). Comparative performance of X ray diffraction and Raman microprobe techniques for the study of carbon materials. Journal of Materials Chemistry, 8: 2875-2879. https://doi.org/10.1039/a805841e

Deines, P. (2004). Carbon isotope effects in carbonate systems. Geochimica at Cosmochimica Acta, 68: 25692679. https://doi.org/10.1016/j.gca.2003.12.002

French, B. (1964). Graphitization of organic material in a progressively metamorphosed Precambrian iron formation. Science, 146: 917-918. https://doi. org/10.1126/science.146.3646.917

García, J. \& Campos, V. (1972). Las rocas paleozoicas en la región del rio Momboy. Boletín de Geología, 5: 2, 796-806.

González de Juana, C.; Iturralde, J. \& Picard, X. (1980). Geología de Venezuela y sus cuencas petrolíferas. Foninves, Caracas, 1030 pp.

Griffin, G. (1967). X-ray diffraction techniques applicable to studies of diagenesis and low rank metamorphism in humic sediments. Journal of Sedimentary Petrology, 37: 1006-1011. https://doi.org/10.1306/ 74D71816-2B21-11D7-8648000102C1865D

Hilchie, L. \& Jamieson, R. (2014). Graphite thermometry in a low-pressure contact aureole, Halifax, Nova Scotia. Lithos, 208-209: 21-33. https:/doi. org/10.1016/j.lithos.2014.08.015

Hoefs, J. (1987). Stable isotope geochemistry, minerals and rocks., Springer-Verlag, Berlin, 241 pp. https:// doi.org/10.1007/978-3-662-09998-8

Hoefs, J. \& Frey, M. (1976). The isotopic composition of carbonaceous matter in a metamorphic profile from the Swiss Alps. Geochimica et Cosmochimica Acta, 40: 945-951. https://doi.org/ 10.1016/0016-7037(76)90143-5

Hollister, L. \& Burruss, R. (1976). Phase equilibria in fluid inclusions from the Khtada metamorphic complex. Geochimica et Cosmochimica Acta, 40: 163175. https://doi.org/10.1016/0016-7037(76)90174-5

Kovisars, L. (1972). Geología de la parte nor-central de los Andes Venezolanos. Boletín de Geología, 5: 817-860.

Kwiecinska, B. \& Petersen, H. (2004). Graphite, semi-graphite, natural coke, and natural char 
classification - ICCP system. International Journal of Coal Geology 57: 99-116. https://doi.org/10.1016/j. coal.2003.09.003

Landis, C. (1971). Graphitization of dispersed carbonaceous materials in metamorphic rocks. Lithos, 14: 215-224. https://doi.org/10.1007/BF00373366

Luque,F; Ortega,L; Barrenechea, J.; Millward, D.; Beyssac, O. \& Huizenga, J. (2009). Deposition of highly crystalline graphite from moderate-temperature fluids. Geology, 37: 275-278. https://oi. org/10.1130/G25284A.1

Luque, F.; Crespo-Feo, E.; Barrenechea, J. \& Ortega, L. (2012). Carbon isotopes of graphite: Implications on fluid history. Geoscience Frontiers, 3: 197-207. https://doi.org/10.1016/j.gsf.2011.11.006

Martínez, M. \& Escobar, M. (2016). Ciencia del Carbón: Geología, Química, Petrografía, Geoquímica, Aplicaciones. Editor Antonio Madrid Vicente, Madrid, 231 pp.

Morikiyo, T. (1986). Hydrogen and carbon isotope studies on the graphite-bearing metapelites in the northern Kiso district of central Japan. Contributions to Mineralogy and Petrology, 94: 165-177. https://doi. org/10.1007/BF00592933

Nabelek, P.; Huff, T. \& Wilke, M. (2002). Carbonic fluid production during regional and contact metamorphism in the Black Hills, USA. Goldschmidt Conference Abstracts, Davos (Switzerland), A543.

Nakamura, D. (1995). Comparison and interpretation of graphitization in contact and regional metamorphic rocks. The Island Arc, 4: 112-127. https://doi. org/10.1111/j.1440-1738.1995.tb00136.x

Nakamura, Y. \& Akai, J. (2013). Microstructural evolution of carbonaceous material during graphitization in the Gyoja-yama contact aureole : HRTEM, XRD and Raman spectroscopic study. Journal of Mineralogical and Petrological Sciences, 108: 131-143. https://doi.org/10.2465/jmps. 120625

Pattison, D. (2006). The fate of graphite in prograde metamorphism of pelites: An example from the Ballachulish aureole, Scotland. Lithos, 88: 85-99. https://doi. org/10.1016/j.lithos.2005.08.006

Satish-Kumar, M. \& Wada, H. (2000). Carbon isotopic equilibrium between calcite and graphite in Skallen Marbles, East Antarctica: evidence for the preservation of peak metamorphic temperatures. Chemical Geology, 166: 173-182. https://doi.org/10.1016/ S0009-2541(99)00189-8
Schubert, C. (1969). Guía de la excursión geológica, región Barinitas-Santo Domingo. IV Congreso Geológico Venezolano, Sociedad Venezolana de Geólogos, Caracas, 272-290.

Tagiri, M. (1981). A measurement of the graphitizing degree by the X-ray Powder Diffractometer. Journal of Japanese Association of Mineralogists, 76: 345352. https://doi.org/10.2465/ganko1941.76.345

Tagiri, M. \& Oba, T. (1986). Hydrothermal syntheses of graphite from bituminous coal at at $0.5-5 \mathrm{kbar}$ water vapor pressure and $300-600^{\circ} \mathrm{C}$. The Journal of the Japanese Association of Mineralogists, Petrologists and Economic Geologists, 81: 260-271. https://doi. org/10.2465/ganko1941.81.260

Tagiri, M.; Yago, Y. \& Tanaka, Y. (2000). Shuffled-cards structure and different $\mathrm{P} / \mathrm{T}$ conditions in the Sanbagawa metamorphic belt, Sakuma-Tenryu area, central Japan. The Island Arc, 9: 188-203. https://doi. org/10.1046/j.1440-1738.2000.00272.X

Török, K. (1992). Cordierite-andalusite-bearing micaschist from the Garbonc-1 borehole (Central Transdanubia, W. Hungary) Geothermo-barometry and fluid inclusion study. European Journal of Mineralogy, 4: 11251136. https://doi.org/10.1127/ejm/4/5/1125

Tracy, R. \& Frost, B. (1991). Phase Equilibria and Thermobarometry of calcareous, ultramafic and mafic rocks and Iron formations. Reviews in Mineralogy and Geochemistry, 26: 207-289.

Turner, F. (1968). Metamorphic petrology. Mc Graw-Hill, New York, 318 pp.

Van Der Lelij, R.; Spikings, R.; Ulianov, A.; Chiaradia, M. \& Mora, A. (2016) Palaeozoic and Early Jurassic history of the north western corner of Gondwana, and implications for the evolution of the Iapetus, Rh. Gondwana Research, 31: 271-294. https://doi. org/10.1016/j.gr.2015.01.011

Wada, H.; Tomita, T.; Matsuura, K.; Iuchi, K.; Ito, M. \& Morikiyo, T. (1994). Graphitization of carbonaceous matter during metamorphism with references to carbonate and pelitic rocks of contact and regional metamorphisms, Japan. Contributions to Mineralogy and Petrology, 118: 217-228. https://doi.org/10.1007/ BF00306643

Wopenka, B. \& Pasteris, J. (1993). Structural characterization of kerogens to granulite-facies graphite : Applicability of Raman microprobe spectroscopy. American Mineralogist, 78: 533-557. 